\title{
Swimming training affects apoptosis-related microRNAs and reduces cardiac apoptosis in mice
}

\author{
Yongcai Zhao and Zhiyong Ma \\ Department of Physical Education, Tangshan Normal University, Hebei, PR China
}

\begin{abstract}
We sought to investigate effects of exercise training on apoptosis-related microRNAs (miRs) and their validated targets, discussing molecular mechanism of the exercise-induced benefit in heart. Male C57BL/6 mice were randomly assigned to three groups: sedentary (SE), exercise training 1 (ET1) and exercise training 2 (ET2). ET1 swam for 8 weeks, once a day and 5 days per week with incremental load. ET2 performed the same work as ET1 and switched to twice a day by the end of the 5th week. In ET2, positive cell rate (\%) tested by TUNEL assay decreased significantly $(p<0.05)$, and the load decreased miR-1 level by $29 \%(p<0.01)$, also increased miR-30b and miR-21 levels by $32 \%(p<0.01)$ and $18 \%(p<0.05)$ respectively. In addition, Bcl-2 expression was increased by $98 \%(p<0.01)$. p53, PDCD4 and Drp-1 expressions were decreased by $45 \%(p<0.01), 6 \%(p>$ $0.05)$ and $36 \%(p<0.01)$ respectively, compared with SE. In ET1, only miR-30b level was increased by $22 \%(p<0.05)$ with a $48 \%$ decrease in $\mathrm{p} 53$ level $(p<0.01)$. Both swimming groups increased Bcl-2/Bax ratio significantly $(p<0.01)$. This study indicated that apoptosis-related miRs and their downstream proteins in heart can be influenced by swimming training that may be responsible for the exercise-induced cardioprotection.
\end{abstract}

Key words: Swimming - Exercise - Heart - MicroRNAs - Apoptosis

\section{Introduction}

The heart is an organ composed of terminally differentiated cardiomyocytes and undergoes cardiac remodeling in response to a variety of stressor. For instance, excessive apoptosis in cardiomyocytes occurs during ischemia-reperfusion (IR), heart failure, and aging (Kitsis and Mann 2005; Kwak et al. 2006), which result in abnormal cardiomyocytes loss. Since cardiomyocytes loss cannot be compensated via efficient cell proliferation, high levels of apoptosis in cardiomyocytes may lead to the pathophysiological disorders. The high level of apoptosis is related to cardiac diseases,

Electronic supplementary material. Supplementary methods. The online version of this article (doi: 10.4149/gpb_2016012) contains supplementary material, which is available to authorized users.

Correspondence to: Yongcai Zhao, Department of Physical Education, Tangshan Normal University, Jian-she north road 156, Tangshan, Hebei province 063000, PR China

E-mail: zyc8256@sina.com such as myocardial infarction, cardiomyopathy and cardiac hypertrophy (Crow et al. 2004).

Thus, it is urgent to develop strategies that result in cardioprotective effects, antagonizing apoptosis involved in cardiomyocytes loss. Numerous approaches to cardioprotection have been investigated, and a wide array of epidemiological studies indicates that regular exercise reduces the risk of death due to an IR insult (Ignarro et al. 2007). Animal experiments also show that consecutive bouts of endurance exercise provide cardioprotection against IR injury and downregulate the activation of the Fas-dependent and mitochondria-dependent apoptotic pathways in hypertensive hearts (Huang et al. 2012; Powers et al. 2014). In addition, regular exercise can decrease the pro-apoptotic protein and increase anti-apoptotic protein in cardiomyocytes that attenuates the extent of apoptosis in physiological condition (Siu et al. 2004).

MicroRNAs (miRs) are small noncoding single stranded RNAs molecules that downregulate their target gene expression. Growing evidence indicates that miRs have distinct expression profiles and function in regulation of gene expression during cardiac cell growth, differentiation, and 
apoptosis (Latronico et al. 2007). Recently, studies indicate that miR-1, -21 and -30 are involved in regulating cardiomyocyte. miR-1 is to be specifically expressed in cardiac muscle and has been certified the pro-apoptosis function by inhibition of Bcl-2 expression (Tang et al. 2009). On the contrary, miR-30 family members can inhibit expression of p53 directly and decrease it's downstream protein level, dynamin-related protein-1 (Drp-1), which initiates the mitochondrial fission program, thereby suppressing apoptosis in heart (Li et al. 2010). miR-21 can bind to the 3'UTR of PDCD4 mRNA and inhibit PDCD4 expression (Asangani et al. 2008), decreasing apoptotic signaling. After initiation of ischemia treatment, miR-21 is upregulated in cardiomyocytes quickly, resulting in suppression of PTEN and downregulation of PDCD4 (Kukreja et al. 2011). Although miRs regulate apoptosis, it remains unclear how they are integrated into the apoptotic program in some pathophysiological conditions. For example, whether miRs are altered during or after exercise in cardiomyocytes, and whether the alterations of miRs affected by exercise contribute to the regulation of apoptosis in heart.

Although it has been recognized that exercise can downregulate apoptosis in cardiomyocytes, the molecular mechanism remains largely unknown and there are no publications reporting the effects of exercise on miRs and their targeted proteins involved in apoptosis of heart. Thus, we established swimming training workload in animal experiments, investigating the effects of exercise on the miR-1, -21 and $-30 b$ expressions and their validated target proteins related to apoptosis. It is a valuable strategy to study the mechanism of exercise-regulated apoptosis for the development of novel approaches in the treatment of cardiovascular diseases.

\section{Materials and Methods}

\section{Experimental design}

Animal experiments were approved by the Research Commission on Ethics of Tangshan Normal University and

Table 1. The primers of miR-1, -21 and $-30 \mathrm{~b}$

\begin{tabular}{ll}
\hline Name & Sequence (5’-3’) \\
\hline mmu-miR-1 F:5’ & ACGCCTGGAATGTAAAGAAGTATG \\
mmu-miR-21 F:5’ & ACACTCCAGCTGGGTAGCTTATCA- \\
& GACTGATG \\
mmu-miR-30b F:5’ & ACACTCCAGCTGGGTGTAAACATC- \\
& CTACACTC \\
miRNA-R:5' & CTCAACTGGTGTCGTGGA \\
U6-F:5' & CTCGCTTCGGCAGCACA \\
U6-R:5' & AACGCTTCACGAATTTGCGT \\
\hline
\end{tabular}

conformed to the Guide for the Care and Use of Laboratory Animals (NIH Publication No. 85-23, revised 1996). C57BL/6 mice (male, 2 months old) were randomly assigned to one of three groups: sedentary (SE), exercise training protocol 1 (ET1) and exercise training protocol 2 (ET2), $n=7$ /group. All animals were housed on a $12 \mathrm{~h}$ light-dark cycle and fed standard mouse chow, and had ad libitum access to water.

\section{Exercise training protocol}

Animals in SE did not do any exercise. Animals in ET1 and ET2 performed increased load swimming training which consisted of swimming sessions for 8 weeks, 5 days/ week. The swimming duration is $30 \mathrm{~min}$ at beginning, and the time was increased by 10 min per week. In the 7 th and 8th week, swimming duration maintained at 90 min. In ET1, animals performed one time a day in whole training period. In ET2, animals performed the same work as ET1 until the end of the fifth week. Then, mice trained twice a day with an interval at least for $6 \mathrm{~h}$ until the end of training. All hearts were harvested $24 \mathrm{~h}$ after the final exercise bout. Samples were quickly frozen in liquid nitrogen and stored at $-80^{\circ} \mathrm{C}$ for miRs, proteins and TUNEL assay.

\section{Detection of miR-1, -21 and -30b by RT-PCR assay}

\section{Total RNA extraction}

Total RNA was isolated according to the manufacturer's directions (Invitrogen). RNA was quantified by BioPhotometer plus to test its purity, $\mathrm{OD}_{260} / \mathrm{OD}_{280}>1.8$.

\section{Real-Time RT-PCR assay}

After cDNA synthesis, quantitative real-time PCR (qPCR) was performed using ABI PRISM 7500 Sequence Detection System (Applied Biosystems) according to the manufacturer's protocol in triplicates. PCR was performed using the RT product and microRNA-specific PCR primers for 40 cycles. The $20 \mu \mathrm{l} \mathrm{PCR}$ included $5 \mu \mathrm{l}$ R product, $10 \mu \mathrm{l}$ (2×) SYBR Green qPCR SuperMix (Invitrogen), $4 \mu \mathrm{l}$ nuclease-free water and $1 \mu \mathrm{l}$ of primers for miRs. The reactions were incubated in a 96 -well optical plate at $50^{\circ} \mathrm{C}-2 \mathrm{~min}$, $95^{\circ} \mathrm{C}-2 \mathrm{~min}$, and then, followed by 40 cycles of $95^{\circ} \mathrm{C}$ for $15 \mathrm{~s}$ and $60^{\circ}$ for $32 \mathrm{~s}$. miRs expressions were normalized by evaluating U6 expression. The threshold cycle (Ct) was set within the exponential phase of the PCR. The target PCR Ct values were normalized by subtracting the U6 Ct value, which provided the $\Delta \mathrm{Ct}$ value. Relative gene expression $=$ $2^{-(\Delta \text { Ctsample- } \Delta \text { Ctcontrol })}$. The primers of miRs used in PCR were shown in Table 1. 


\section{Detection of apoptosis}

The heart tissue was immersion-fixed in 10\% neutral formalin and embedded in paraffin. Serial sections of $4-\mu \mathrm{m}$ thicknesses were prepared. Apoptotic cells were identified by terminal deoxynucleotidyl transferase dUTP nick end labeling (TUNEL) using an apoptosis detection kit according to the manufacturer's protocol (Cat.No. G7130, Promega). Detailed materials are described in the Supplementary Methods.

\section{Western blotting analysis}

Western blotting analysis was performed using standard procedures as described previously (Duan et al. 2012). Quantification was performed in the linear absorption range by computerized densitometry using Scion Image software. GAPDH levels of heart tissues were used to normalize the results. Detailed materials are described in the Supplementary Methods.

\section{Statistical analysis}

All data are presented as mean \pm SD. For relative gene expression, the mean value of the sedentary group is defined as 1 . Statistical analysis was performed by one-way ANOVA for the evaluation of the data. The Pearson correlation analysis was used to analyze the correlations between parameters. $p<$
0.05 was considered significant. All statistics were computed using PASW Statistics 18.0 software.

\section{Results}

\section{Exercise training downregulates the apoptosis in} cardiomyocytes

The first step is to test whether exercise training affect apoptosis in cardiomyocytes. The results obtained from TUNEL assay indicated that the rate of apoptotic cells in ET2 (1.45 $\pm 0.39 \%, p<0.01)$ was significantly lower than that in SE $(2.26 \pm 0.55 \%)$ and ET1 $(2.41 \pm 0.56 \%)$. In addition, although the mice in ET1 undertook exercise training for 8 weeks, the apoptosis rate in their hearts did not alter significantly compared with SE $(p>0.05)$ (Fig. 1). Together, it seems that relative heavy load swimming training in ET2 can result in significant downregulation of apoptosis in heart cells tested by TUNEL assay.

miRs related to apoptosis of cardiomycoyte are changed by exercise training

Studies have shown that miRs control diverse aspects of heart disease (Divakaran and Mann 2008). Previous studies led us to consider whether long-term swimming training can affect apoptosis-related miRs. We tested the alterations
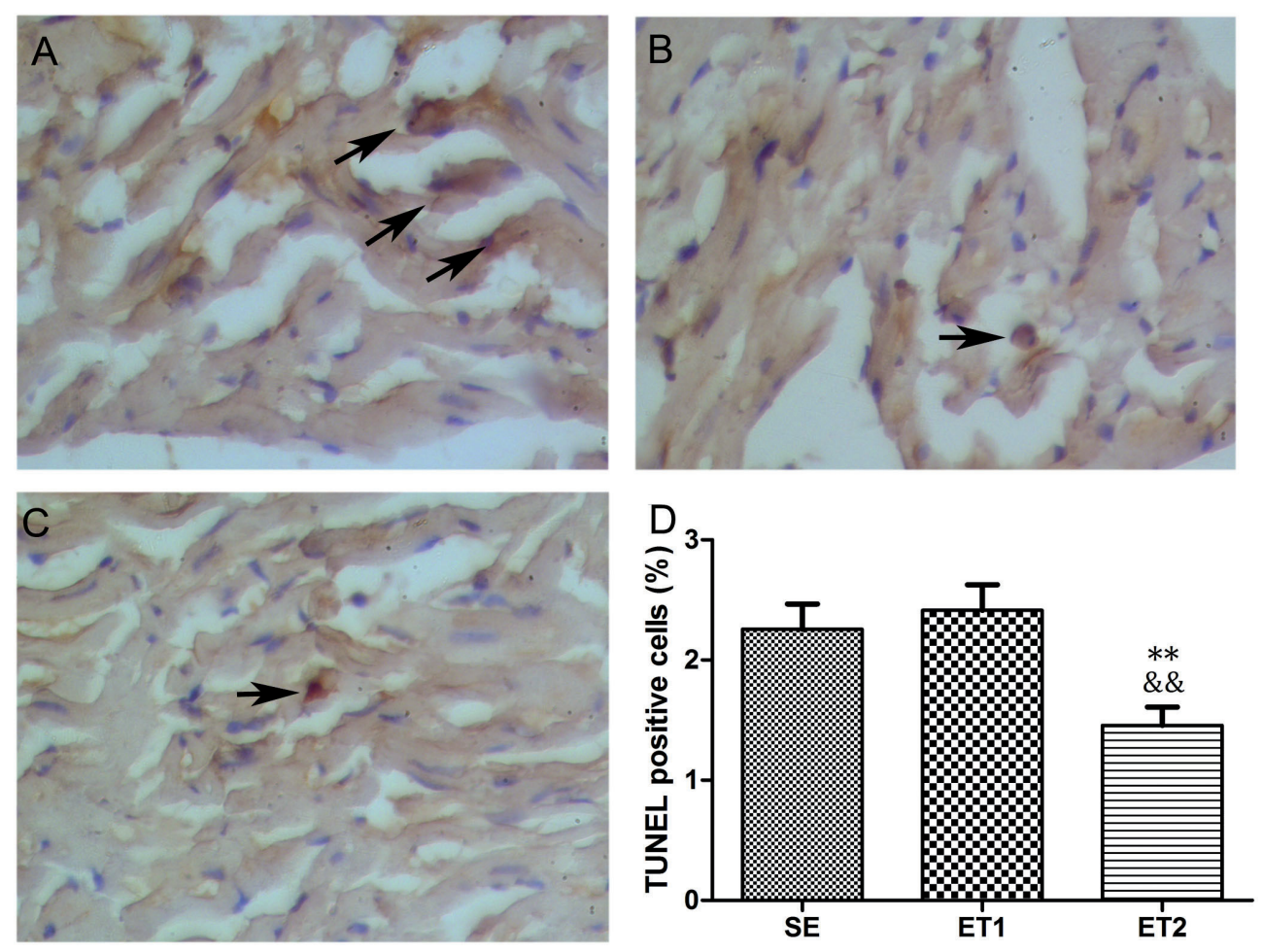

Figure 1. Effects of swimming training on the apoptosis of cardiomyocytes by TUNEL assay. Representative images of ventricular myocardium sections in SE (A), ET1 (B) and ET2 (C). Black arrows point to the apoptotic cells. Quantitative analysis of apoptosis by TUNEL assay (D). Values are mean \pm SD. $n=7 /$ group, ${ }^{* *} p<0.01 \mathrm{vs}$. SE, ${ }^{\& \&} p<0.05$ vs. ET1. SE, sedentary group; ET1, exercise training 1 group; ET2, exercise training 2 group. 
A
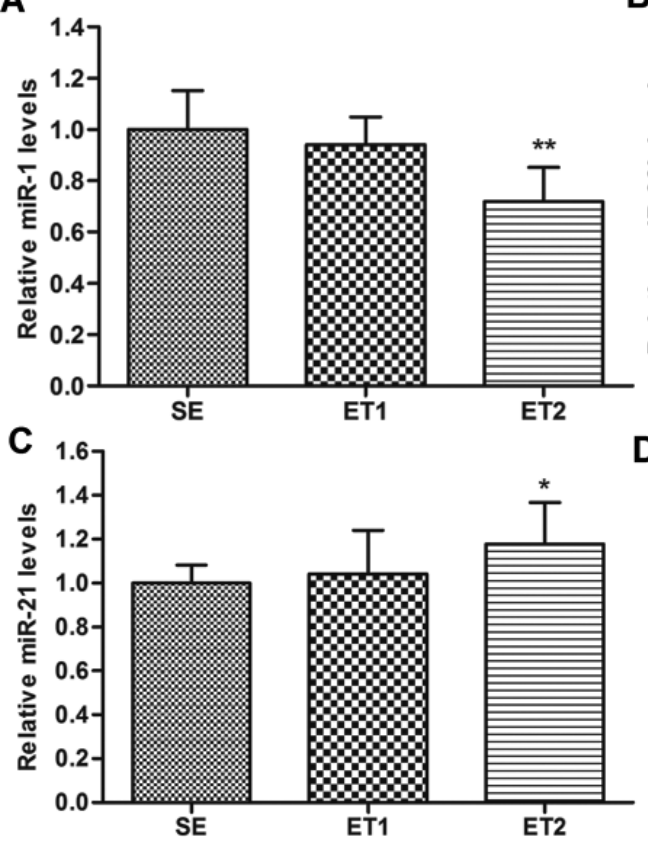

B
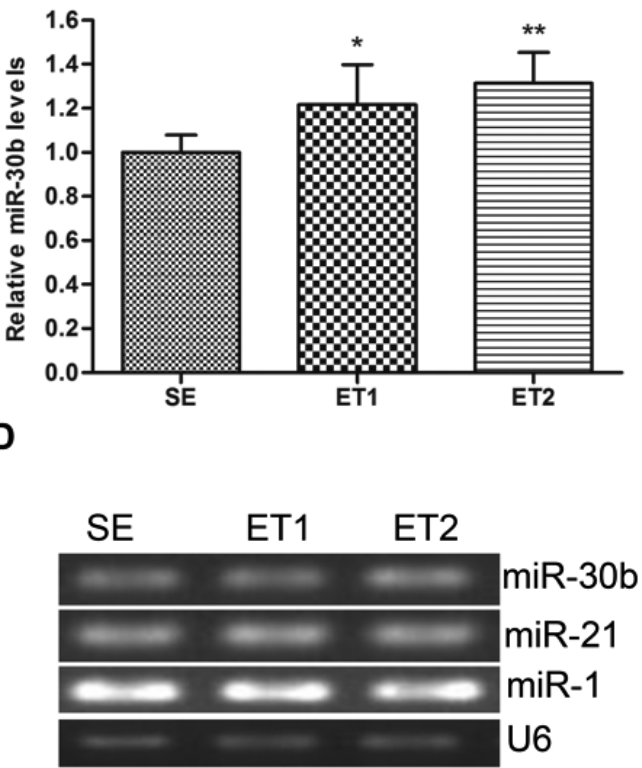

Figure 2. miRs involved in apoptotic regulation were changed by exercise training. miR-1 was down-regulated in ET1 and ET2 and only the decrease in ET2 had statistical significance $(\mathbf{A})$; miR-30b levels were both increased significantly in ET1 and ET2 (B); miR-21 had a slight elavation in ET1 and a significant elavation in ET2 (C). Gel electrophoresis image of RT-PCR products of miRs (D). Values are mean \pm SD. $n=7$ /group, ${ }^{\star} p<0.05$ $v s$. SE, ${ }^{* *} p<0.01 v s$. SE. For abbreviations, see Fig. 1. of miR-1, miR-21 and miR-30b in response to swimming training. As shown in Fig. 2A, miR-1 levels in ET1 and ET2 were both downregulated, and the levels were reduced by $6 \%$ in ET1 $(p>0.05)$ and $29 \%$ in ET2 $(p<0.01)$ compared with SE. Fig $2 \mathrm{~B}$ showed significant differences in miR-30b among three groups. miR-30b levels were increased by $22 \%$ in ET1 $(p<0.05)$ and $32 \%$ in ET2 $(p<0.01)$ compared with SE. In contrast to the sensibility of miR-30b, miR-21 levels were increased by $4 \%$ in ET1 $(p>0.05)$ and $18 \%$ in ET2 $(p<$ 0.05) compared with SE (Fig. 2C), which implied that relative heavy load training in present study could influence miR-1 and miR-21 apparently.
A

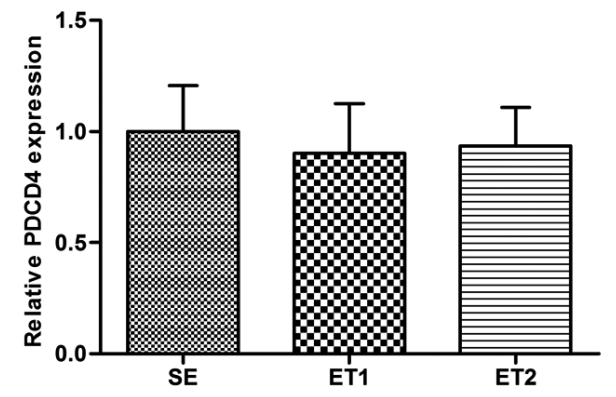

C

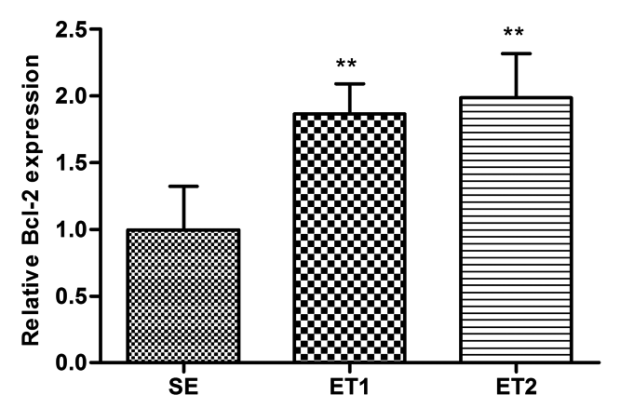

B

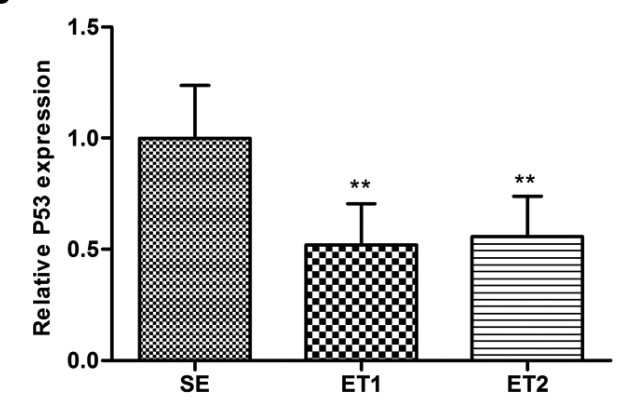

SE ET1 ET2

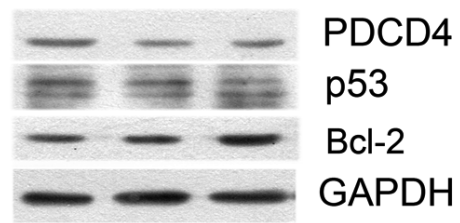

Figure 3. Effects of swimming training on miRs-targeted proteins in apoptotic regulation of cardiomyocytes. The expression of PDCD4 was decreased slightly by exercise training (A), and a remarkable decease in p53 was also observed (B). In contrast, Bcl-2 was increased in exercise protocols (C). Representative blots of PDCD4, p53 and Bcl-2 (D). Values are mean \pm SD. $n=7 /$ group, ${ }^{* *} p<0.01$ $v s$. SE. For abbreviations, see Fig. 1. 
The changes of miRs-targeted proteins involved in apoptosis of cardiomycoyte

Bcl-2, PDCD4 and p53 expressions can be inhibited directly by miR-1, -21 and -30 b respectively (Asangani et al. 2008; Tang et al. 2009; Li et al. 2010). Western blot assay demonstrated that PDCD4 expressions were downregulated in ET1 and ET2 compared with SE (Fig. 3A). But there were no statistical significances among the three groups ( $9 \%$ and $6 \%$ reduction in ET1 and ET2 respectively, $p>0.05)$. p53 expressions in ET1 and ET2 were reduced by $48 \%(p<0.01)$ and $45 \%(p<0.01)$ in ET1 and ET2 compared with SE respectively (Fig 3B). In contrast to the reduction of PDCD4 and p53 expressions, swimming training significantly increased the Bcl-2 expressions in ET1 and ET2 (86\% and 98\% increase in ET1 and ET2 respectively, $p<0.01$ ) (Fig. 3C). Altogether, pro-apoptotic proteins expressions such as PDCD4 and p53 can be decreased, but Bcl-2 that antagonizes apoptosis can be increased by swimming training. p53 and Bcl-2 are more sensitive than PDCD4 in response to swimming training.

The changes of pro-apoptotic proteins involved in apoptosis of cardiomycoyte

Drp1 which is induced by p53 is a GTPase that causes scission of the mitochondrial outer membrane, resulting in fission of mitochondrial tubules into fragments. It is also responsible for cytochrome $\mathrm{c}$ release and caspase activation (Frank et al. 2001). Bax, a member of the Bcl-2 proapoptotic family, can also be induced by p53. In response to stress activation, Bax forms a homodimer and releases cytochrome c from the mitochondria, which results in caspase-9 activation (Miyashita and Reed 1995). We are interested in the alteration of Drp-1 and Bax controlled by p53. Drp-1 levels were reduced by $17 \%$ in ET1 $(p>0.05)$ and $36 \%$ in ET2 $(p<0.01)$ compared with SE (Fig. 4 A). Bax levels were downregulated greatly by swimming training. Reduction in ET1 and ET2 reached to $38 \%(p<0.01)$ and $60 \%(p<0.01)$ compared with SE respectively (Fig. 4B). $\mathrm{Bcl}-2$ and Bax represent the anti- and pro-apoptotic properties in mitochondria-dependent pathway, and the Bcl-2/ Bax ratio were increased by 2 folds in ET1 and nearly 4 folds in ET2 compared with SE $(p<0.01)$ (Fig. 4 C). Although significant downregulation of apoptosis by TUNEL assay was not observed in ET1, our results indicates that ET1 and ET2 both attenuate apoptotic signals estimated by apoptotic proteins expressions.

\section{Correlations among different apoptotic regulators in present study}

To further clarify the relations between the proteins and miRs in our study, we used the Pearson coefficient of correlation to analyze their correlations. Consistent with our hypothesis, undergoing different workloads in mice, miR-1 and Bcl-2 appeared a significant negative correlation $(\mathrm{R}=-0.543, p<$ $0.05)$, and the same result was observed between miR-30b and $\mathrm{p} 53(\mathrm{R}=-0.633, p<0.01)$. Interestingly, a special discovery in present study was that miR-21 and PDCD4 had no significant negative correlation due to the low correlation coefficient $(\mathrm{R}=-0.062, p>0.05)$. Additionally, we studied the relations among these proteins in present research.
A
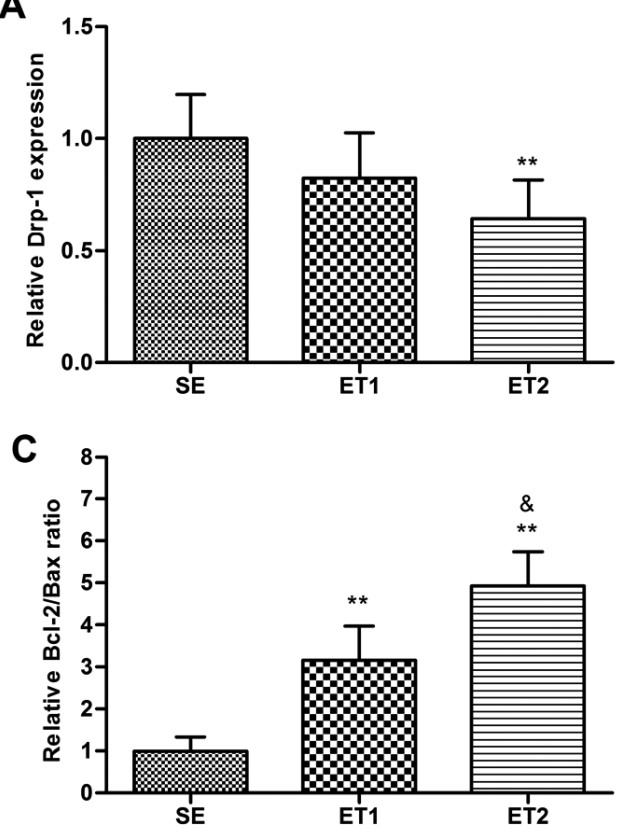

B

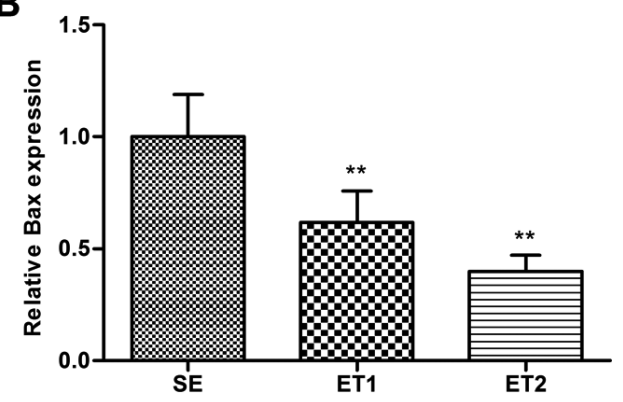

D

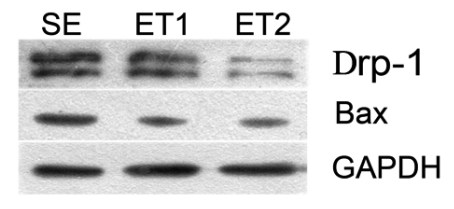

Figure 4. Effects of swimming training on the pro-apoptotic proteins involved in intrinsic apoptosis pathway. The expression of Drp-1 was decreased in ET1 and ET2 (A). A similar decreased change in Bax was induced by the two trainings (B). The Bcl-2/Bax ratio was increased in ET1 and ET2 (C). Representative blots of Drp-1 and Bax (D). Values are mean \pm SD. $n=7 /$ group, ${ }^{* *} p<0.01$ vs. SE, $\& p<0.05 v s$. ET1. For abbreviations, see Fig. 1. 
A positive correlation was found between $\mathrm{p} 53$ and Drp-1 ( $\mathrm{R}$ $=0.738, p<0.01)$, p53 and Bax also had the same relation $(\mathrm{R}=0.52, p<0.01)$.

\section{Discussion}

The heart may undergo an increase in apoptosis during ischemia-reperfusion, heart failure, and aging (Chen et al. 2009; Duan et al. 2012; Fannin et al. 2013). On the other hand, high apoptosis in heart is believed to contribute to impaired contractile function, cardiomyopathy and heart disease (Masri and Chandrashekhar 2008; Favaloro et al. 2012). Therefore, developing a strategy that results in a cardioprotection is important. Much evidence contends that exercise is cardioprotective. First, previous studies confirmed that exercise reduces the risk of heart diseases in pathological models. For instance, fewer Fas-dependent and mitochondria-dependent apoptotic proteins were found in exercise group in spontaneously hypertensive rats (Lee et al. 2006; Huang et al. 2012). A second, in vitro experiments, the exercise-induced cardioprotection is mediated through mitochondrial adaptations, because isolated mitochondria resist ROS and IR-induced damage (Kavazis et al. 2008; Lee et al. 2012). We want to investigate whether exercise leads to the same benefit in physiological condition of healthy individual. A study from Kwak et al. (2006) demonstrated that exercise training can reduce age-induced apoptosis in rats. The present study tested the effects of swimming training on the apoptosis of heart in adult mice, consistent with previous observations; our data showed that TUNEL positive cell rate in ET2 was decreased significantly, while mice in ET1 did not create the same result. The current data suggested that swimming training could lead to relatively low level cardiac apoptosis in adult mice. The reason why ET1 failed to produce the protective effect may be the exercise intensity was relatively weak.

Understanding of the cellular mechanisms responsible for this cardioprotection remains incomplete. Heart is energetically-demanding tissue requiring continuous production of ATP from mitochondria which occupy up to $25 \%$ of cell volume ( $\mathrm{Li}$ et al. 2012). The merger of adverse events may orchestrate opening of the mitochondrial permeability transition pore, leading to cell death through necrosis or apoptosis (Kung et al. 2011). Thus, we mainly focused on mitochondria-dependent pathway in apoptosis. miRs are about 22 nucleotides long and act as negative regulators of gene expression by inhibiting mRNA translation or promoting mRNA degradation (Lee et al. 2003). miRs can regulate cardiac function, including the conductance of electrical signals, heart muscle contraction, heart growth and morphogenesis (Chien 2007). miR-1 was suggested to participate in apoptosis, and $\mathrm{Bcl}-2$ was also confirmed to be a direct target of miR-1 later (Tang et al. 2009). In current study, we evaluated changes of miR-1 and Bcl-2 in response to different swimming training protocols. The results revealed that miR-1 expressions were downregulated in ET1 and ET2, and the decreased extent in ET2 had statistical significance. In contrary to the decrease in miR-1, the Bcl-2 expressions were upregulated in the two training groups. In ET1, the significant increase of Bcl-2 level was not consistent with the change of miR-1, which suggested other upstream regulators affect $\mathrm{Bcl}-2$ expression more effectively than miR-1. The Bcl-2 level also had a significant negative correlation with miR-1 level, which suggests, the exercise induced cardioprotection in current study may be partly attributed to the changes of miR-1 and Bcl-2.

The miR-30 family consists of five members, miR-30a through -30e. Recent studies identified that miR-30 family also mediates the mitochondria-dependent apoptosis in heart. A study from Shi et al. reported that apoptosisassociated genes were highly enriched among the predicted targets of miR-30 family, and the total protein and phosphorylation expressions of $\mathrm{p} 53$ were inhibited by miR-30 in podocytes (Shi et al. 2013). A study from Li et al. (2010) also proved that miR-30a and miR-30b directly targets p53 in heart. miR-30b appeared to be more sensitive to ROS than the other family members (Haque et al. 2012). Additionally, after fractionating the heart of mice into nonmyocyte and cardiomyocyte populations, miR-30b other than miR-30a was substantially enriched in cardiomyocytes (Ikeda et al. 2009). Thus, we study the change of miR-30b upon exercise training. p53, a down stream target of miR-30b, can initiate apoptosis by transcriptionally regulating the gene products of the pro-apoptotic proteins. For instance, it can activate Bax, which translocates from the cytosol to mitochondria and induces the release of apoptotic proteins (Miyashita and Reed 1995). Mitochondria constantly undergo fusion and fission, which are necessary for the maintenance of organelle fidelity. Abnormal mitochondrial fission is involved in the initiation of apoptosis, which requires Drp-1, a GTPase induced by $\mathrm{p} 53$ that causes scission of the mitochondrial outer membrane. Drp-1 accumulates on mitochondria and mediates dramatic mitochondrial fission contributing to caspase activation in cardiomyocytes (Wang et al. 2011). We evaluated miR-30b-p53-downstream proteins axis in the current study. The results showed that miR-30b level was upregulated, while p53, Drp-1 and Bax levels were downregulated significantly in both ET1 and ET2. It is of note that ET1 also resulted in significant increase in miR-30b and decrease in pro-apoptotic proteins, especially in p53 and Bax. This implied that moderate or relative heavy load swimming training could result in beneficial effects in view of the miR-30b-p53-downstream proteins axis. The further analysis also indicated there were significant negative correlation between miR-30b and p53, 
positive correlation between 553 and Drp-1. Together, our study suggested that miR-30b-p53- downstream proteins axis is responsible for the cardioprotective effects induced by exercise training.

miR-21 is found to be an anti-apoptotic regulator (Buscaglia and Li 2011). Transfection with adenovirus expressing miR-21 reduced cell apoptosis in heart, decreased myocardial infarction size, and improved left ventricular remodeling (Dong et al. 2009). Cheng et al. (2009) found miR-21 downregulated PDCD4 expression, and the protective effect of miR-21 on cell death was inhibited by overexpression of PDCD4. Another study confirmed that miR-21 directly binds to PDCD4 and inhibit PDCD4 expression by the luciferase assay (Cheng et al. 2010). PDCD4 is a critical mediator for cancer cell apoptosis, PDCD4 was overexpressed, then, cardiomyocyte apoptosis induced by ischemia-reperfusion was aggravated consequently (Cheng et al. 2010). We tested the expressions of miR-21 and PDCD4. miR-21 seemed to be less susceptible than miR$30 \mathrm{~b}$, only the increase in ET2 had statistical significance. Change in miR-21 meant that the increase in miR-21 may be a reason for the relatively low level of cardiac apoptosis in ET2. Interestingly, we did not observe remarkable decrease in PDCD4 expressions in ET1 and ET2, which suggested swimming training can not affect the expression of PDCD4 apparently. The relation between miR-21 and PDCD4 was assessed, and there was no significant correlation. Despite the notable increased miR-21 level in ET2, the PDCD4 level in ET1 and ET2 did not change with statistical significance. It is possible that some regulating factors induced by exercise interfere with the relation between miR-21 and PDCD4. Taken together, miR-21 and PDCD4 appear more sustainable upon the exercise training than miR-1 and $\mathrm{miR}-30 \mathrm{~b}$ regulatory pathways.

Of note that the two swimming training resulted in different effects on apoptosis in the heart. Although the load in ET1 affected some proteins levels significantly, the significant decrease in apoptosis evaluated by TUNEL assay was not observed, which meant that the molecular changes in ET1 is not enough to induce the expected effects. In contrast, load in ET2 both affected miR-1-Bcl-2, miR-30b-p53 pathways apparently. In addition, apoptotic cell rate in ET2 decreased significantly. Together, ET2 other than ET1 can lead to more notable cardioprotection in the present work. Altogether, our study suggested that the changes of miRs and their downstream proteins induced by swimming training participate in reducing the apoptotic level in hearts.

Acknowledgments. This study was supported by natural science foundation of Tangshan Normal University (2016B04).

Conflict of interest. The authors have no conflict of interest to declare.

\section{References}

Asangani I. A., Rasheed S. A., Nikolova D. A., Leupold J. H., Colburn N. H., Post S., Allgayer H. (2008): MicroRNA-21 (miR21) post-transcriptionally down-regulates tumor suppressor Pdcd4 and stimulates invasion, intravasation and metastasis in colorectal cancer. Oncogene 27, 2128-2136

http://dx.doi.org/10.1038/sj.onc.1210856

Buscaglia L. E., Li Y. (2011): Apoptosis and the target genes of miR-21. Chin. J. Cancer. 30, 371-380 http://dx.doi.org/10.5732/cjc.30.0371

Chen L., Gong Q., Stice J. P., Knowlton A. A. (2009): Mitochondrial OPA1, apoptosis, and heart failure. Cardiovasc. Res. 84, 91-99 http://dx.doi.org/10.1093/cvr/cvp181

Cheng Y., Liu X., Zhang S., Lin Y., Yang J., Zhang C. (2009): MicroRNA-21 protects against the $\mathrm{H} 2 \mathrm{O} 2$-induced injury on cardiac myocytes via its target gene PDCD4. J. Mol. Cell. Cardiol. 47, 5-14 http://dx.doi.org/10.1016/j.yjmcc.2009.01.008

Cheng Y., Zhu P., Yang J., Liu X., Dong S., Wang X., Chun B., Zhuang J., Zhang C. (2010): Ischaemic preconditioning-regulated miR-21 protects heart against ischaemia/reperfusion injury via anti-apoptosis through its target PDCD4. Cardiovasc. Res. 87, 431-439 http://dx.doi.org/10.1093/cvr/cvq082

Chien K. R. (2007): Molecular medicine: microRNAs and the telltale heart. Nature 447, 389-390 http://dx.doi.org/10.1038/447389a

Crow M. T., Mani K., Nam Y. J., Kitsis R. N. (2004): The mitochondrial death pathway and cardiac myocyte apoptosis. Circ. Res. 95, 957-970 http://dx.doi.org/10.1161/01.RES.0000148632.35500.d9

Divakaran V., Mann D. L. (2008): The emerging role of microRNAs in cardiac remodeling and heart failure. Circ Res. 103, 1072-1083

http://dx.doi.org/10.1161/CIRCRESAHA.108.183087

Dong S., Cheng Y., Yang J., Li J., Liu X., Wang X., Wang D., Krall T. J., Delphin E. S., Zhang C. (2009): MicroRNA expression signature and the role of microRNA-21 in the early phase of acute myocardial infarction. J. Biol. Chem. 284, 29514-29525 http://dx.doi.org/10.1074/jbc.M109.027896

Duan X., Ji B., Wang X., Liu J., Zheng Z., Long C., Tang Y., Hu S. (2012): Expression of microRNA-1 and microRNA-21 in different protocols of ischemic conditioning in an isolated rat heart model. Cardiology 122, 36-43 http://dx.doi.org/10.1159/000338149

Fannin J., Rice K. M., Thulluri S., Arvapalli R. K., Wehner P., Blough E. R. (2013): The Effects of aging on indices of oxidative stress and apoptosis in the female fischer 344/Nnia X bown Norway/ BiNia rat heart. Open. Cardiovasc. Med. J. 29, 113-121 http://dx.doi.org/10.2174/1874192401307010113

Favaloro B., Allocati N., Graziano V., Di Ilio C., De Laurenzi. V. (2012): Role of apoptosis in disease. Aging 4, 330-349 http://dx.doi.org/10.18632/aging.100459

Frank S., Gaume B., Bergmann-Leitner E. S., Leitner W. W., Robert E. G., Catez F., Smith C. L., Youle R. J. (2001): The role of dynamin-related protein $\mathbf{1}$, a mediator of mitochondrial fission, in apoptosis. Dev. Cell. 1, 515-525 http://dx.doi.org/10.1016/S1534-5807(01)00055-7 
Haque R., Chun E., Howell J. C., Sengupta T., Chen D., Kim H. (2012): MicroRNA-30b-mediated regulation of catalase expression in human ARPE-19 cells. PLoS One 7, e42542 http://dx.doi.org/10.1371/journal.pone.0042542

Huang C. Y., Yang A. L., Lin Y. M., Wu F. N., Lin J. A., Chan Y. S., Tsai F. J., Tsai C. H., Kuo C. H., Lee S. D. (2012): Anti-apoptotic and pro-survival effects of exercise training on hypertensive hearts. J. Appl. Physiol. 112, 883-891 http://dx.doi.org/10.1152/japplphysiol.00605.2011

Ignarro L. J., Balestrieri M. L., Napoli C. (2007): Nutrition, physical activity, and cardiovascular disease: an update. Cardiovasc. Res. 73, 326-340 http://dx.doi.org/10.1016/j.cardiores.2006.06.030

Ikeda S., He A., Kong S. W., Lu J., Bejar R., Bodyak N., Lee K. H., Ma Q., Kang P. M., Golub T. R., Pu W. T. (2009): MicroRNA-1 negatively regulates expression of the hypertrophyassociated calmodulin and Mef2a genes. Mol. Cell. Biol. 29, 2193-2204 http://dx.doi.org/10.1128/MCB.01222-08

Kavazis A. N., McClung J. M., Hood D. A., Powers S. K. (2008): Exercise induces a cardiac mitochondrial phenotype that resists apoptotic stimuli. Am. J. Physiol. Heart. Circ. Physiol. 294, H928-935 http://dx.doi.org/10.1152/ajpheart.01231.2007

Kitsis R. N., Mann D. L. (2005): Apoptosis and the heart: a decade of progress. J. Mol. Cell. Cardiol. 38, 1-2 http://dx.doi.org/10.1016/j.yjmcc.2004.11.008

Kukreja R. C., Yin C., Salloum F. N. (2011): MicroRNAs: new players in cardiac injury and protection. Mol. Pharmacol. 80, $558-564$ http://dx.doi.org/10.1124/mol.111.073528

Kung G., Konstantinidis K., Kitsis R. N. (2011): Programmed necrosis, not apoptosis, in the heart. Circ. Res. 108, 1017-1036 http://dx.doi.org/10.1161/CIRCRESAHA.110.225730

Kwak H. B., Song W., Lawler J. M. (2006): Exercise training attenuates age-induced elevation in $\mathrm{Bax} / \mathrm{Bcl}-2$ ratio, apoptosis, and remodeling in the rat heart. FASEB J. 20, 791-793 http://dx.doi.org/10.1096/fi.05-5116fje

Latronico M. V., Catalucci D., Condorelli G. (2007): Emerging role of microRNAs in cardiovascular biology. Circ. Res. 101, $1225-1236$ http://dx.doi.org/10.1161/CIRCRESAHA.107.163147

Lee Y., Min K., Talbert E. E., Kavazis A. N., Smuder A. J., Willis W. T., Powers S. K. (2012): Exercise protects cardiac mitochondria against ischemia-reperfusion injury. Med. Sci. Sports. Exerc. 44, 397-405 http://dx.doi.org/10.1249/MSS.0b013e318231c037

Lee Y., Ahn C., Han J., Choi H., Kim J., Yim J., Lee J., Provost P., Rådmark O., Kim S., Kim V. N. (2003): The nuclear RNase
III Drosha initiates microRNA processing. Nature 425, 415-419 http://dx.doi.org/10.1038/nature01957

Lee Y. I., Cho J. Y., Kim M. H., Kim K. B., Lee D. J., Lee K. S. (2006): Effects of exercise training on pathological cardiac hypertrophy related gene expression and apoptosis. Eur. J. Appl. Physiol. 97, 216-224 http://dx.doi.org/10.1007/s00421-006-0161-5

Li J., Donath S., Li Y., Qin D., Prabhakar B. S., Li P. (2010): miR30 regulates mitochondrial fission through targeting p53 and the dynamin-related protein-1 pathway. PLoS Genet. 6, e1000795 http://dx.doi.org/10.1371/journal.pgen.1000795

Li Q., Zhou L.Y., Gao G. F., Jiao J. Q., Li P. F. (2012): Mitochondrial network in the heart. Protein Cell 3, 410-418 http://dx.doi.org/10.1007/s13238-012-2921-9

Masri C., Chandrashekhar Y. (2008): Apoptosis: a potentially reversible, meta-stable state of the heart. Heart Fail. Rev. 13, 175-179

http://dx.doi.org/10.1007/s10741-007-9069-3

Miyashita T., Reed J. C. (1995): Tumor suppressor p53 is a direct transcriptional activator of the human bax gene. Cell 80, 293-299 http://dx.doi.org/10.1016/0092-8674(95)90412-3

Powers S. K., Smuder A. J., Kavazis A. N., Quindry J. C. (2014): Mechanisms of exercise-induced cardioprotection. Physiology $29,27-38$ http://dx.doi.org/10.1152/physiol.00030.2013

Shi S., Yu L., Zhang T., Qi H., Xavier S., Ju W., Bottinger E. (2013): Smad2-dependent downregulation of miR-30 is required for TGF- $\beta$-induced apoptosis in podocytes. PLoS One 8, e75572 http://dx.doi.org/10.1371/journal.pone.0075572

Siu P. M., Bryner R. W., Martyn J. K., Alway S. E. (2004): Apoptotic adaptations from exercise training in skeletal and cardiac muscles. FASEB J. 18, 1150-1152 http://dx.doi.org/10.1096/fj.03-1291fje

Tang Y., Zheng J., Sun Y., Wu Z., Liu Z., Huang G. (2009): MicroRNA-1 regulates cardiomyocyte apoptosis by targeting Bcl-2. Int. Heart J. 50, 377-387 http://dx.doi.org/10.1536/ihj.50.377

Wang J. X., Jiao J. Q., Li Q., Long B., Wang K., Liu J. P., Li Y. R., Li P. F. (2011): miR-499 regulates mitochondrial dynamics by targeting calcineurin and dynamin-related protein-1. Nat. Med. 17, 71-78 http://dx.doi.org/10.1038/nm.2282

Received: February 4, 2016

Final version accepted: March 30, 2016

First published online: September 9, 2016 


\title{
Swimming training affects apoptosis-related microRNAs and reduces cardiac apoptosis in mice
}

\author{
Yongcai Zhao and Zhiyong Ma \\ Department of Physical Education, Tangshan Normal University, Hebei, PR China
}

\section{Supplementary Methods}

\section{Detection of apoptosis}

The heart tissue was immersion-fixed in $10 \%$ neutral formalin and embedded in paraffin. Serial sections of $4-\mu \mathrm{m}$ thicknesses were prepared. Apoptotic cells were identified by terminal deoxynucleotidyl transferase dUTP nick end labeling (TUNEL) using an apoptosis detection kit according to the manufacturer's protocol (Cat.No. G7130, Promega). Briefly, the tissue sections were dewaxed twice in xylene, 5 minutes each time, and rehydrated in decreasing concentrations of ethanol (100\%, 95\%, 85\%, 70\%, 50\%), 3 minutes each time. After that, sections were immersed in $0.85 \% \mathrm{NaCl}$ for 5 minutes and in PBS for 5 minutes respectively. The sections were fixed and washed, then, added with $100 \mu$ l Equilibration Buffer for 5 minutes. $100 \mu \mathrm{l}$ of TdT reaction were added to the tissue sections on the slides and the sections were incubated for 60 minutes at $37^{\circ} \mathrm{C}$ in a humidified chamber. Then, the reaction was stopped and slides were immersed in $2 \mathrm{X}$ SSC for 15 minutes. After washing and then immersing slides in $0.3 \%$ hydrogen peroxide for 5 minutes, $100 \mu$ Streptavidin HRP (diluted 1:500 in PBS) was added to slides which were incubated for 30 minutes at room temperature. After washing slides in PBS, sections were added $100 \mu \mathrm{DAB}$ (prepared immediately prior to use). Then, slides were immersed in deionized water for washing. The percentage of myocytes with DNA nick end labeling was analyzed by counting the cells exhibiting brown nuclei at $\times 20$ magnification in 10 randomly chosen fields (Olympus, CX41), total 1000 cells were counted in each section (4 sections for each sample, average percentage was the sample's rate of apoptotic cells).

\section{Western blotting analysis}

Total proteins were extracted from the frozen heart tissue. The samples were placed in RIPA buffer (Beyotime Inst. Biotech, China), homogenized, incubated on ice for $20 \mathrm{~min}$ and then centrifuged at $12,000 \times g$ for $20 \mathrm{~min}$. Samples were subjected to SDS-PAGE in polyacrylamide gels (6-15\%) depending on the protein molecular weight. After electrophoresis, proteins were transferred to the polyvinylidene fluoride membrane (Cat.No. IPVH00010, Millipore). Ponceau S red (Sigma) was used to confirm equal loading and transfer of proteins to the membrane in each lane. The blot membrane was then incubated in a blocking buffer ( $5 \%$ non-fat dry milk, $10 \mathrm{mM}$ Tris- $\mathrm{HCl}$ (pH 7.6), $100 \mathrm{mM} \mathrm{NaCl}$, and $0.1 \%$ Tween 20) for $2 \mathrm{~h}$ at room temperature and then incubated overnight at $4^{\circ} \mathrm{C}$ with specific antibody. These antibodies included $\mathrm{p} 53$ polyclonal antibody, Bcl-2 polyclonal antibody, Bax polyclonal antibody and PDCD4 polyclonal antibody (Santa Cruz Biotechnology, CA, USA, 1:1,000 dilution); Dynamin-related protein-1 (Drp-1) polyclonal antibody (Cell Signaling Tech, MA, USA, 1:1,000 dilution); HRP-conjugated monoclonal GAPDH antibody (KangChen Bio-tech, China, 1:10,000 dilution). Immunodetection of the primary antibody was carried out with peroxidase-labeled secondary antibodies and enhanced chemiluminescence reagents (Engreen Biosystem Co. Ltd, China) were used to visualize the autoradiogram. Quantification was performed in the linear absorption range by computerized densitometry using Scion Image software. GAPDH levels of heart tissues were used to normalize the results. 